\title{
Oropharyngeal flora and individual susceptibility to neisserial infection
}

\author{
H YOUNG,* A B HARRIS,* AND D H H ROBERTSON† \\ From the *Department of Bacteriology, Edinburgh University Medical School, and the +University \\ Department of Venereology, Royal Infirmary, Edinburgh
}

SUMMARY $\beta$-haemolytic streptococci were isolated from throat swabs from $49(10 \cdot 5 \%)$ of 466 patients undergoing cultural examination for gonorrhoea. Although $\beta$-haemolytic streptococci were isolated more frequently from patients with genital or anorectal gonorrhoea $(15 \cdot 9 \%)$ than from those without $(9 \cdot 2 \%)$, the difference was not statistically significant. When groupable (A, B, $C$, or $G$ ) and other (non-A, $-B,-C$, or $-G) \beta$-haemolytic streptococci were analysed separately, a statistically significant association between non-A, $-\mathrm{B},-\mathrm{C}$, or $-\mathrm{G}$ streptococci and gonococci was observed but not between groupable $\beta$-haemolytic streptococci and gonococci.

\section{Introduction}

Since the preliminary observations of Willcox et al, ${ }^{1}$ it has now been convincingly demonstrated that Neisseria meningitidis is isolated at least twice as often from the throats of patients with genital gonorrhoea than from patients without gonorrhoea and that Neisseria gonorrhoeae is isolated from a genital site approximately twice as often from patients with meningococci in the throat than from patients without. $^{2-4}$ To date, only one study ${ }^{5}$ has found no such association between genital gonorrhoea and oropharyngeal carriage of meningococci.

$\varnothing$ degaard and Gedde-Dahl ${ }^{4}$ observed that these results could indicate either that there is individual susceptibliliy to neisserial infection or that there is simply a difference in the behaviour patterns of patients who contract gonorrhoea, the conduct of the latter making them not only more likely to be infected with $N$ gonorrhoeae but possibly more exposed to the acquisition of $N$ meningitidis as well. We made a similar suggestion ${ }^{3}$ and also postulated that if these results on the carriage of neisseriae merely reflect the association of intimate behaviour with the exchange of flora then oropharyngeal carriage of other "marker" organisms should show a similar correlation with genital gonorrhoea.

This paper reports our findings with $\beta$-haemolytic streptococci as marker organisms.

Address for reprints: Dr H Young, Department of Bacteriology Edinburgh University Medical School, Teviot Place, Edinburgh EH8 9AG

Received for publication 1 February 1980

\section{Patients and methods}

Unselected patients who attended the Department of Venereology at the Royal Infirmary, Edinburgh, for the first time during the months of September, October, November, and December 1979 were included in the survey. The sites sampled as part of the diagnostic routine, and the culture and identification methods for gonococci and meningococci, were as described previously. ${ }^{3}$

\section{OROPHARYNGEAL CULTURE}

An additional throat swab was taken from each patient and received at the laboratory within four hours. On receipt, each specimen was streaked on to blood agar medium (Gibco blood agar base $+5 \%$ human blood) and modified New York City (MNYC) medium. ${ }^{6}$ Both media were incubated at $37^{\circ} \mathrm{C}$ in an aerobic atmosphere enriched with $10 \% \mathrm{CO}_{2}$. After incubation for 24 hours a representative colony from those plates with $\beta$-haemolytic colonies on the blood agar medium was inoculated into Todd-Hewitt broth and subcultured on to a blood agar plate. After overnight incubation the Todd-Hewitt broth culture was checked by Gram-staining and streptococci were grouped by a coagglutination method (Phadebact streptococcus test; Pharmacia Diagnostics, Uppsala, Sweden) with reagents specific for groups A, B, C, and $\mathrm{G}$. The blood agar plate served as a purity check and confirmed the $\beta$-haemolytic nature of the isolate. MNYC plates were examined at 24 and 48 hours and any oxidase-positive Gram-negative diplococci were identified by standard methods. ${ }^{3}$ 
STATISTICAL METHOD

Statistical analysis of the results was made by the $\chi^{2}$ method with Yates's correction.

\section{Results}

N GONORRHOEAE

Specimens from 466 patients (290 men and 176 women) were examined. $N$ gonorrhoeae was isolated by culture from genital or anorectal sites of 88 $(18 \cdot 9 \%)$ patients ( 51 men and 37 women). $N$ gonorrhoeae was also isolated from the throat of two of these patients and from the throat only of one additional patient.

\section{$\beta$-HAEMOLYTIC STREPTOCOCCI}

$\beta$-haemolytic streptococci $(\beta$-HS) were isolated from throat swabs of $49(10.5 \%)$ patients (36 men and 13 women). Of the $49 \beta$-HS isolates, two were group $A$, six group $B$, nine group $C$, three group $G$, and 29 were not $A, B, C$, or $G$.

$\beta$-HS (16 groupable and 19 non- $A,-B,-C$, or $-G$ ) were isolated from $35(9 \cdot 2 \%)$ of 378 patients with negative genital and ano-rectal culture results for $N$ gonorrhoeae. $\beta$-HS (four groupable and 10 non-A, $-B$, -C, or $-G)$ were isolated from $14(15 \cdot 9 \%)$ of 88 patients with positive genital or ano-rectal culture results for gonococci. Gonococci were isolated from $74(17 \cdot 8 \%)$ of 417 patients with negative culture results for $\beta$-HS and from $14(28 \cdot 6 \%)$ of 49 patients from whom $\beta$-HS were cultured.

\section{ASSOCIATION OF ORGANISMS}

Although $\beta$-HS were isolated more frequently from patients with than from those without gonorrhoea, and gonococci were isolated more frequently from patients with than those without oropharyngeal $\beta$-HS, the association of each organism with the other was not statistically significant $\left(\chi_{1}^{2} 2 \cdot 7 ; 0 \cdot 2>\right.$ $\mathrm{P}>\mathbf{0} \cdot \mathbf{1}$ ).

When the groupable and non- $A,-B,-C$, or $-G$ $\beta$-HS are analysed separately the following associations are obtained. Excluding the 29 patients with non- $A,-B,-C$, or $-G \beta-H S$, groupable $\beta$-HS were isolated from $16(4 \cdot 5 \%)$ of the 359 patients without gonorrhoea and from four $(5 \cdot 1 \%)$ of the 78 patients with gonorrhoea. Excluding the 20 patients with groupable $\beta$-HS, non- $A,-B,-C$, or $-G \beta$-HS were isolated from $19(5 \cdot 3 \%)$ of the 362 patients without gonorrhoea and from $10(13 \cdot 5 \%)$ of the 84 patients with gonorrhoea. The association of non- $A,-B,-C$, or -G $\beta$-HS with gonococci was statistically significant $\left(\chi_{1}^{2} 3 \cdot 9 ; 0.05>\mathrm{P}>0.02\right)$ whereas there was no statistically significant association between groupable $\beta$-HS and gonococci $\left(\chi_{1}^{2} 0 \cdot 001 ; \mathrm{P}>0.9\right)$.
N MENINGITIDIS

Meningococci were isolated from $17(3 \cdot 7 \%)$ of the 466 throat swabs plated in the laboratory: meningococci were isolated from seven $(8 \%)$ of the 88 patients with gonorrhoea and from $10(2 \cdot 7 \%)$ of the 378 patients without gonorrhoea. The association of gonococci and meningococci was statistically significant $\left(\chi_{1}^{2} 4 \cdot 3 ; 0 \cdot 05>\mathrm{P}>0 \cdot 02\right)$.

Oropharyngeal specimens from 233 patients were examined for meningococci both by direct plating and by throat swabs sent to the laboratory: meningococci were isolated from $55(23 \cdot 6 \%)$ patients by direct plating compared with only $10(4 \cdot 3 \%)$ from swabs. The association of gonococci and meningococci in the 233 patients on whose throat specimens direct plating was done was significant $\left(\chi_{1}^{2}\right.$ $6 \cdot 9 ; \mathrm{P}<0 \cdot 01)$.

\section{Discussion}

Unfortunately, the question of whether or not the reported association of genital gonorrhoea and oropharyngeal carriage of meningococci ${ }^{1-4}$ is the result of individual susceptibility to neisserial infection or is attributable to behavioural factors cannot be answered from our results. This is partly due to the complication that arises since the association of the groupable $\beta$-HS (A, B, C, and $G$ ) in patients with and without genital or anorectal gonorrhoea differs significantly from that of the non-A, $-B,-C$, or $-G$ $\beta$-haemolytic streptococci. Streptococci belonging to groups $A, B, C$, and $G$ are the ones most often associated with human streptococcal infection. ${ }^{7}$ Therefore the division between groupable and nongroupable $\beta$-HS made in this study is not without justification.

Our finding of an association between non-A, -B, $-C$, or $-G \beta$-HS and gonorrhoea could possibly be explained as a result of quantitative differences in the oropharyngeal flora secondary to altered host resistance after infection with $N$ gonorrhoeae. There are inherent difficulties in expressing what is essentially a quantitative situation by the qualitative parameters of growth or no growth on a culture plate. This is particularly true when it is borne in mind that over $90 \%$ of organisms collected on the swab will adhere so tightly that they will not be released on inoculation of the culture plate. ${ }^{8}$

A suitable method of quantitative bacteriological sampling will probably be required before the factors governing the association of gonococci and meningococci can be elucidated by the aid of marker oropharyngeal organisms. It is also possible that both individual susceptibility and benavioural factors contribute to the observed association making the specific contribution of each difficult to assess. 
The different behaviour of the non-A, -B, -C, or $-G$ and groupable $\beta$-HS suggests that differences might also exist between certain sero-groups and non-groupable meningococci with reference to their association with gonococci. This possibility is under investigation at present. Whether or not different sub-populations of meningococci could explain the failure of Noble et $a l^{5}$ to demonstrate an association between gonococci and meningococci is open to speculation.

Thanks are extended to the staff of the Department of Venereology, Edinburgh Royal Infirmary, for sending clinical specimens and to Dr P W Ross and Professor J G Collee for their helpful advice during the preparation of the paper.

\section{References}

1. Willcox RR, Spencer RC, Ison C. Which Neisseria? Br J Vener Dis 1977; 53:394-7.

2. Rufli Th. Which Neisseria? Br J Vener Dis 1978;54:352.

3. Young $H$, Harris AB, Robertson DHH. Individual susceptibility to neisserial infection? Br J Vener Dis 1979;55: 188-90.

4. Ødegaard K, Gedde-Dahl TW. Frequency of simultaneous carriage of Neisseria gonorrhoeae and Neisseria meningitidis. Br J Vener Dis 1979; 55:334-5.

5. Noble RC, Cooper RM, Miller BR. Pharyngeal colonisation by Neisseria gonorrhoeae and Neisseria meningitidis in black and white patients attending a venereal disease clinic. $B r J$ Vener Dis 1979; 55: 14-19.

6. Young $\mathrm{H}$. Cultural diagnosis of gonorrhoea with modified New York City (MNYC) medium. Br J Vener Dis 1978; 54:36-40.

7. Pollock HM, Dahlgren BJ. Distribution of streptococcal groups in clinical specimens with evaluation of bacitracin screening. Appl Microbiol 1974; 26:141-3.

8. Ross PW. The isolation of Streptococcus pyogenes from throat swabs. J Med Microbiol 1977; 10:69-76. 